Research Article

\title{
Investigating Photochromic Behavior of Organic Dyes in Solution Form using Multilevel Factorial Design
}

\author{
Najiah Nadir ${ }^{1}$ Zaharah Wahid ${ }^{2, *}$, Amir Akramin Shafie ${ }^{1}$, Farah B. Ahmad ${ }^{3}$, \\ Mat Tamizi Zainuddin ${ }^{4}$
}

\begin{abstract}
${ }^{1}$ Department of Mechatronics Engineering, Faculty of Engineering, International Islamic University Malaysia, P. O. Box 10, 50728 Kuala Lumpur, Malaysia.

${ }^{2}$ Department of Science in Engineering, Faculty of Engineering, International Islamic University Malaysia, P. O. Box 10, 50728 Kuala Lumpur, Malaysia.

${ }^{3}$ Department of Biotechnology Engineering, Faculty of Engineering, International Islamic University Malaysia, Kuala Lumpur, Malaysia.
\end{abstract}

${ }^{4}$ Industrial Centre of Innovation (ICI) Biomedical, SIRIM Industrial Research (SIR), SIRIM Berhad, Lot 34, Jalan Hi-Tech 2/3, Kulim Hi-Tech Park, 09000 Kulim, Kedah, Malaysia.

Received: $2^{\text {nd }}$ March 2021; Revised: $2^{\text {nd }}$ April 2021; Accepted: $5^{\text {th }}$ April 2021

Available online: $8^{\text {th }}$ April 2021; Published regularly: June 2021

\section{Abstract}

Photochromic compounds, namely spiropyran, spirooxazine, and naphthopyran, have received much attention, because of their high potential applications in various industrial fields. The aim of this study is to understand the behavior of three photochromic dyes in solution form via statistical approach. The types of dyes and solvents were screened using multilevel factorial design. From the analysis of variance results, it was found that the types of dyes and solvents used as well as their interaction have significant effects on the absorbance and photostability. The naphthopyran compound displayed highest change in absorbance intensity, followed by spiropyran and spirooxazine, when dissolved in isopropanol separately. However, the spirooxazine is the most photostable dye compared to naphthopyran and spiropyran, with ethanol as the solvent.

Copyright (C) 2021 by Authors, Published by BCREC Group. This is an open access article under the CC BY-SA License (https://creativecommons.org/licenses/by-sa/4.0).

Keywords: Photochromism; spiropyran; spirooxazine; naphthopyran; factorial design; photostability

How to Cite: N. Nadir, Z. Wahid, A.A. Shafie, F.B. Ahmad, M.T. Zainuddin (2021). Investigating Photochromic Behavior of Organic Dyes in Solution Form using Multilevel Factorial Design. Bulletin of Chemical Reaction Engineering \& Catalysis, 16(2), 353-365 (doi:10.9767/bcrec.16.2.10496.353-365)

Permalink/DOI: https://doi.org/10.9767/bcrec.16.2.10496.353-365

\section{Introduction}

Photochromism is a part of photochemistry as it involves a reversible change in color of certain substances upon light irradiation [1]. The most well-known application of photochromic materials is the photochromic lens for optical glasses that darken on exposure to UV light. The most commercialized photochromic dyes are

* Corresponding Author.

Email: zaharahwahid@iium.edu.my (Z. Wahid); spiropyrans, spirooxazines, and naphthopyrans [2]. The application of photochromic dyes is extensive which include biomedicine, textiles, thermoplastic, ophthalmic lenses, chemical sensing and surface coatings [2-4]. In biomedicine, photochromic dyes are used in the photoactivation control of fluorescence imaging, drug delivery and biomacromolules; on-demand cell attachment; and photopharmacological chemotherapy [3]. Spiropyran-functionalized polydiacetylene (SFPDA) vesicles were investigated in the detection of cyanide anions $\left(\mathrm{CN}^{-}\right)$that are 
based on colorimetric and fluorometric measurement, in which spiropyrans were used as the receptors for $\mathrm{CN}^{-}$detection based on the nucleophilic addition of $\mathrm{CN}^{-}$that will result in a clear optical change [5]. Sahoo and Kumar reported on the use of spirooxazines for the optical detection of $\mathrm{Fe}^{3+}$ in aqueous solution [6]. As spirooxazines are the photoreversible molecules that oscillate between two different states of closed and open form, the open form with its more polarized character can bind with metal ions especially trivalent metal ion $\mathrm{Fe}_{3}$ [6]. Spiropyran and naphthopyran photochromic dyes were incorporated into polypropylene fibres by melt extrusion for the production of fibres that showed better photostability in polypropylene than in screen-printed textiles [7].

The first aim of this paper is to investigate the behaviour of photochromic organic dyes by observing the absorption spectra and fading kinetic in organic solvents of ethanol and isopropanol. The second aim of this paper is to determine the photochromic dye with the highest absorbance and photostability in solution form, using multilevel factorial design. The photostability or fatigue resistance must be considered and improved because it is the most formidable drawback for photochromic material which reduce their commercialization and practical applications [8-10].

Islam et al. investigated the performance and photofatigue behavior of spirooxazines in ethanol, where maximum deep blue coloration due to active photochromism of spirooxazines was achieved at 6\% UV irradiation power [11]. Nevertheless, existing photochromic studies have focused on the individual parameter while ignoring the interaction between the parameters. It is essential to detect whether the parameters interact with each other or not because there are cases where the interaction is significant even though the individual parameters are insignificant [12-14]. There are no studies that have investigated the interaction between absorption spectra and fading kinetic of photochromic dyes in organic solvents statistically.

The use of a statistical approach allows efficient estimation of the effect of each parameter and also any possible interaction between the parameters using the smallest number of experiments [15]. The multilevel factorial design is capable of producing an experimental design based on the combination of different independent factors at different levels, i.e. one factor at three levels and another factor at two levels, and also identify which factors have significant effects on the response [16].

\section{Materials and Methods}

\subsection{Sample Preparation}

There were three types of photochromic dyes utilised in this study, which are 1,3,3trimethylindolinonaphthospirooxazine (SO), 3,3-diphenyl-3H-naphtho[2,1-b]pyran (NP), and $1^{\prime}, 3^{\prime}$ - dihydro-1' ${ }^{\prime}, 3^{\prime}, 3^{\prime}$ - trimethyl-6nitrospiro[ $2 H$-1-benzopyran-2, $2^{\prime}$ - $(2 H)$-indole] (SP) as shown in Scheme 1. The SO and NP were purchased from Tokyo Chemical Industry and SP from Sigma-Aldrich, respectively. For solvents, absolute ethanol (EtOH) (99\%) was obtained from HmbG Chemicals and isopropyl alcohol or isopropanol (IPA) (70\%) from Systerm. In photolithography room, the SO, NP, and SP dyes were dissolved individually in ethanol and isopropanol at a concentration of 1 $\mathrm{mM}$. The photochromic solutions were stirred at room temperature (RT) for $1 \mathrm{~h}$ and kept in the dark for $24 \mathrm{~h}$. The solutions were then transferred into $10 \mathrm{~mm}$ quartz cuvette prior to UV irradiation.

\subsection{Characterizations}

The absorbance and fading kinetic measurements were performed according to the method described by Nadir et al. $[17,18]$. The dyes were irradiated with UV lamp (Efos Acticure A4000) at an intensity of $0.5 \mathrm{~mW} / \mathrm{cm}^{2}$. The absorption

naphtopyrans
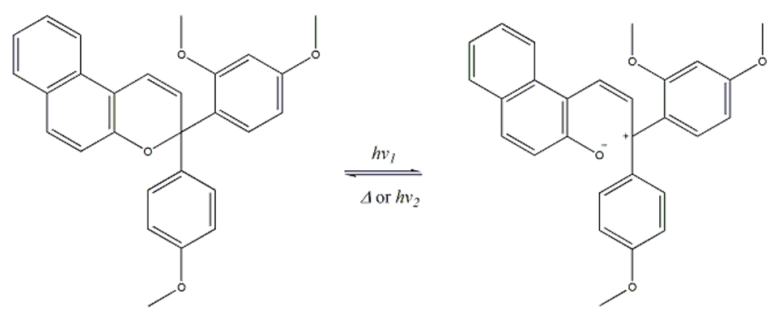

spirooxazines
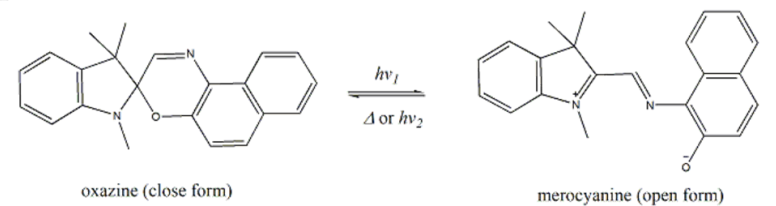

spiropyrans

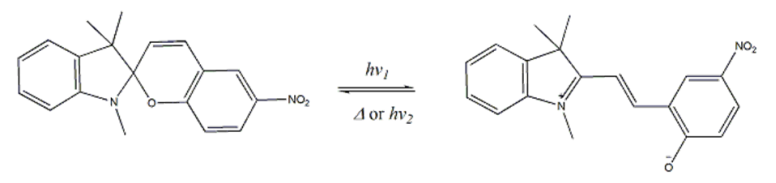

Scheme 1. Photochromic mechanism of naphthopyrans, spirooxazines and spiropyrans [11,39-41]. 
spectra were measured prior to and after UV irradiation by using UV-Vis spectrometer (OceanOptics USB4000) equipped with xenon lamp (OceanOptics PX-2). The absorbance was expressed as a percentage, with the solvent as blank. When any of the sampling variables is changed, a new reference spectrum must be stored.

The change in absorbance intensity was calculated by determining the absorbance prior to and after UV irradiation, which is as follows:

$$
\triangle A=\frac{A_{0}-A_{i}}{A_{0}} \times 100 \%
$$

where $A_{0}$ is the absorbance at time zero (after irradiation) and $A_{i}$ is the absorbance before irradiation.

For fading kinetic measurement, the spectrometer was set at maximum wavelength $\left(\lambda_{\max }\right)$ of the colored forms of the dyes. The dyes were irradiated with UV light and the decrease in absorbance was recorded against time. The fading kinetic of most photochromic dyes fits the first-order exponential function. Thus, the time evolution of the absorbance after UV irradiation, $A(t)$, can be expressed as:

$$
A(t)=\left(A_{0}-A_{i}\right) e^{-k t}+A_{i}
$$

where $A_{0}$ is the absorbance at time zero (after irradiation), $A_{i}$ is the absorbance before irradiation, $k$ is the first-order rate constant for the fading kinetic, and $t$ is the time. All of these were measured at the maximum wavelength, $\lambda_{\max }[19]$.

Reorganizing Equation (2) by moving all the variables for absorbance $\left(A_{i}\right.$ and $\left.A_{0}\right)$ to the left side:

$$
\frac{A(t)-A_{i}}{A_{0}-A_{i}}=e^{-k t}
$$

The natural log was placed at both sides to remove the exponent:

$$
\ln \left(\frac{A(t)-A_{i}}{A_{0}-A_{i}}\right)=\ln \left(e^{-k t}\right)
$$

The right side of the equation was simplified using logarithmic rule:

$$
\ln \left(\frac{A(t)-A_{i}}{A_{0}-A_{i}}\right)=-k t(\ln (e))
$$

By using $\ln (e)=1$, the right side was further simplified:

$$
\ln \left(\frac{A(t)-A_{i}}{A_{0}-A_{i}}\right)=-k t
$$

In order to find $k$, Equation (6) was constructed to fit the equation of a straight line on a graph. The graph of $\ln \left[\left(A(t)-A_{i}\right) /\left(A_{0}-A_{i}\right)\right]$ versus $t$ was plotted and the slope of the subsequent straight line represents the value of $-k$. From this, the half-life time, $t_{1 / 2}$, of the photochromic dye is calculated using Equation (7).

$$
t_{1 / 2}=\frac{\ln 2}{k}
$$

where $t_{1 / 2}$ is actually the time required for the maximum absorbance $\left(A_{0}-A_{i}\right)$ at the $\lambda_{\max }$ of the merocyanine form to decrease to its half value $\left(\left(A_{0}-A_{i}\right) / 2\right)$ following the removal of activating light $[2,20,21]$. The fading rate constant is inversely related to the half-life time.

\subsection{Multilevel Factorial Design}

The multilevel factorial design was selected to study the in-depth relationship of photostability behavior for the inspected dyes within the investigated solvents with different polarity. The behavior of these dyes was observed in two solvents of different polarity because the solvent polarity influences the photochromic behavior. The dye concentration and irradiation time were fixed at $1 \mathrm{mM}$ and $15 \mathrm{~s}$, respectively.

The multilevel factorial design design $(3 \times 2$, replication $=2$ ) generated 12 experimental runs. The absorbance increment was measured daily for 14 days. The mean $(M(\triangle A b s))$ and standard deviation $(S D(\triangle A b s))$ of absorbance change were calculated. This standard deviation was set as the main aspect to observe the dye photostability. Natural logarithmic transformation was applied to model the variability of the standard deviation [16,22]. Hence, the value of natural log was computed and opted as the response. The design was replicated twice to examine the main effects of each factor as well as their interaction effect on the response [16,23]. Also, the sensitivity of the analysis will be improved as the number of experimental runs increases [22].

A multiple regression model was developed using the experimental results to find the relationship between the dye photostability to the types of dye and solvent. The developed model was verified statistically.

\subsection{Software}

SpectraSuite software was employed to measure the absorption spectra and fading kinetic as it can control the Ocean Optics USB spectrometer and support a real-time measurement [18]. Meanwhile, the design of experiments was planned and analysed using the statistical software package Minitab® 16.2.0. 


\section{Results and Discussion}

\subsection{Absorption Spectra}

Upon UV irradiation, all the three dyes in ethanol and isopropanol showed a color change as illustrated in Figure 1. The colorless SO and NP turned blue and orange, respectively, while the very light pink of SP became brighter, indicating that the dyes exhibit mechanism of opened structure from oxazine (for spirooxazines) and pyran (for naphthopyran and spiropyrans) mode to merocyanine mode. This reaction suggested that all the three dyes undergo photoisomerization in the investigated solvents (Scheme 1). On exposure to UV irradiation, the photoisomerization of spirooxazines leads to cleavage of $\mathrm{C}-\mathrm{O}$ oxazine isomer and subsequently the formation of ring-opened merocyanine isomer that is blue in color $[4,11]$. The col-
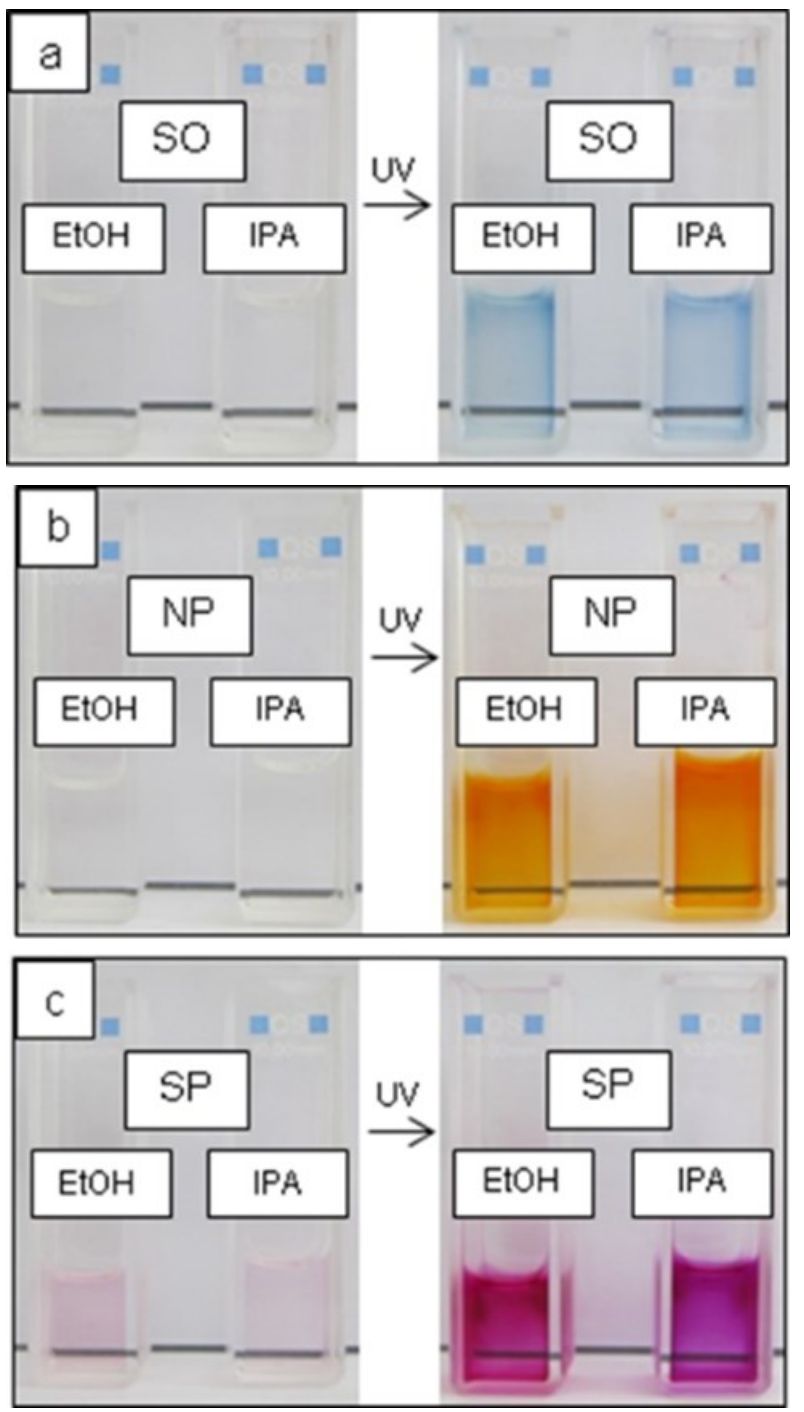

Figure 1. Photocolouration of SO, NP, and SP before and after UV irradiation in ethanol and isopropanol. or faded away once the UV light source is removed due to reverting back of merocyanine to oxazine- and pyran-based structures. Merocyanine isomers that mainly consisted of quinoidal and zwitterionic forms, represent an equilibrium state [4]. Therefore, all three dyes display positive or normal photochromic behavior [24,5].

SO was colorless in both solvents prior to UV irradiation, thus gave nearly zero absorbance intensities as depicted in Figure 2(a). After $15 \mathrm{~s}$ of irradiation, the change in absorbance intensities $(\triangle A)$ went up to $0.108 \%$ for isopropanol (IPA) and $0.098 \%$ for ethanol (EtOH). SO in isopropanol demonstrated higher absorbance increase. The absorption peak was detected at $608 \mathrm{~nm}$ for isopropanol and $613 \mathrm{~nm}$ for ethanol. This designates that SO shows positive solvatochromism as the peak shifted to-
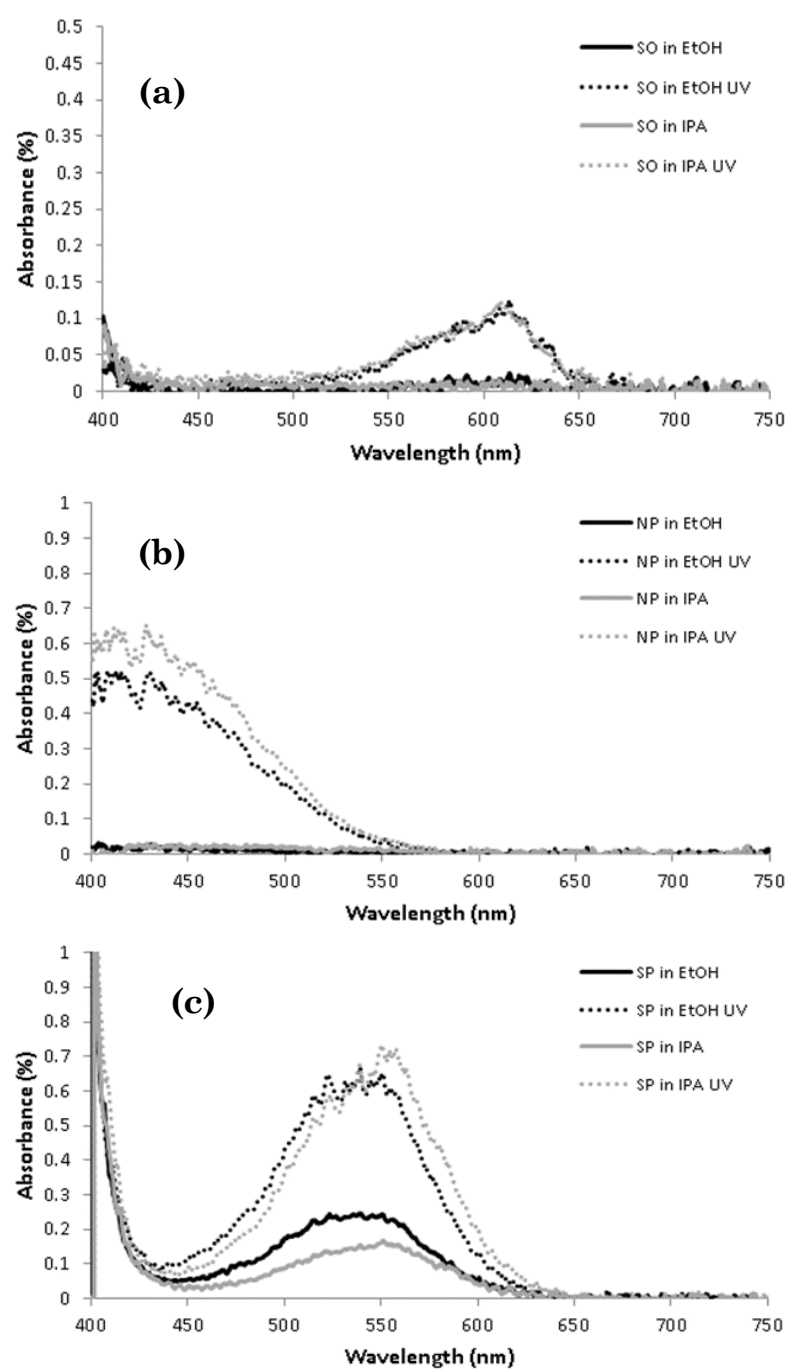

Figure 2. Change in absorbance intensities $(\triangle A)$ spectra of (a) SO, (b) NP and (c) SP in ethanol and isopropanol before and after $15 \mathrm{~s}$ of UV irradiation, at $1 \mathrm{mM}$ of dye concentration. 
wards a longer wavelength (bathochromic or red shift) with increasing solvent polarity [2628]. The change in absorbance intensities $(\Delta A)$ spectra in Figure 2(b) implied that the colorless NP has greater $\Delta A$ in isopropanol (0.604\%) compared to ethanol $(0.487 \%)$, similar to SO. Also, NP dye unveils positive solvatochromism because the maximum absorption band moved bathochromically from $428 \mathrm{~nm}$ to $430 \mathrm{~nm}$ as the solvent polarity increases [29].

Figure 2(c) illustrates the absorption spectra of SP in isopropanol and ethanol. Initially, SP displayed a light pink color in both solvents; hence it gave $\Delta A$ values of $0.162 \%$ in isopropanol and $0.243 \%$ in ethanol. The values then increased to $0.713 \%$ and $0.639 \%$, respectively, after irradiation. SP in isopropanol revealed a higher intensity increase, like SO and NP. The maximum wavelength was shifted to a shorter wavelength (hypsochromic or blue shift) in more polar solvent, from $550 \mathrm{~nm}$ in isopropanol to $538 \mathrm{~nm}$ in ethanol. Therefore, SP is a negative solvatochromic compound $[21,26,30]$.

\subsection{Fading Kinetic}

The fading or bleaching kinetic represents the stabilization of the merocyanine or opened form. When a photochromic dye is more stable
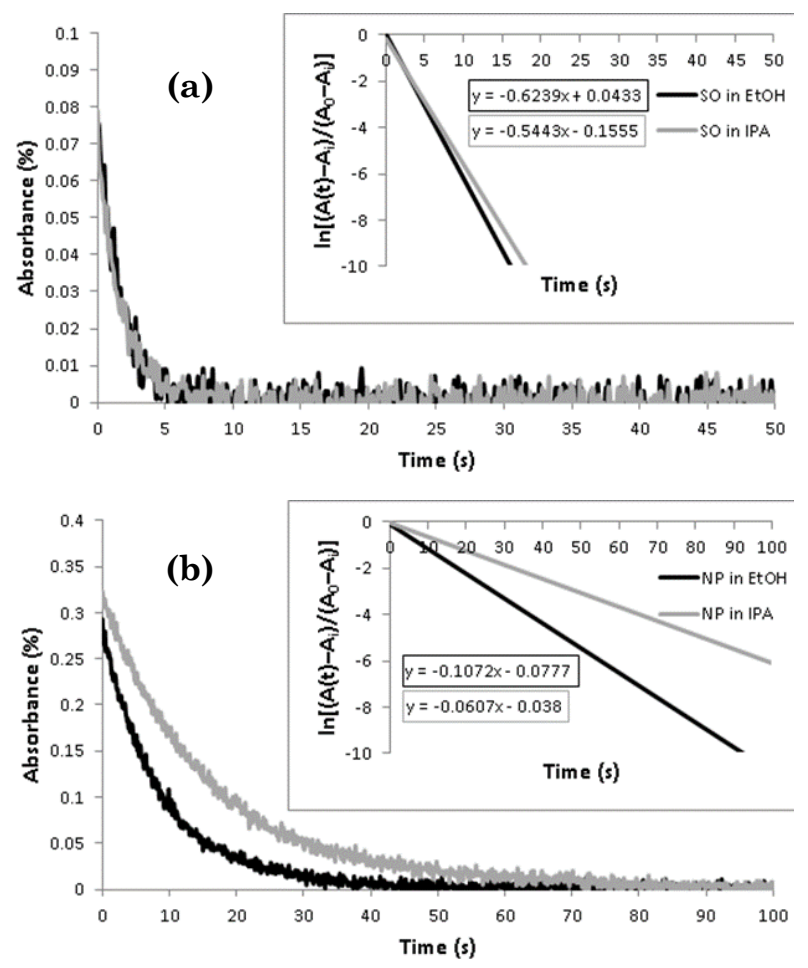

in its opened form than closed form, it will take a longer time to fade and thus the fading rate constant will decrease [31,32]. In solution form, the reverse reaction or fading kinetic of the photochromic dyes is quite well-fitted to the exponential decay function and usually fits firstorder kinetic [33,34].

As seen in Figure 3(a), the fading kinetics of SO in ethanol and isopropanol were almost similar. Both curves follow the first-order of exponential decay function. The time taken for $\Delta \mathrm{A}$ to become zero is around $5 \mathrm{~s}$ for $\mathrm{SO}$ in both solvents. The fading rate constant of SO in ethanol $\left(k=0.6239 \mathrm{~s}^{-1}\right)$ is slightly larger than SO in isopropanol $\left(k=0.5443 \mathrm{~s}^{-1}\right)$, which indicates that the merocyanine form of SO is less stable in ethanol. This is because the stability of the merocyanine form decreases as the fading rate constant increases. The degradation was faster in more polar solvent due to merocyanineoxygen interaction that produced a polar intermediate, which would be stabilized by a polar solvent with a larger dielectric constant [10].

It is evident in Figure 3(b) that the fading kinetics of NP in ethanol and isopropanol are different from each other. With lower $\Delta A$, NP in ethanol took $40 \mathrm{~s}$ to completely fade, while NP in isopropanol took a longer time of $70 \mathrm{~s}$. $\mathrm{NP}$ in ethanol has a higher fading rate constant $\left(k=0.1072 \mathrm{~s}^{-1}\right)$ compared to NP in isopropanol $\left(k=0.0607 \mathrm{~s}^{-1}\right)$. Thus, the opened form of NP is more stable in isopropanol. A similar observation was discovered when NP was dissolved separately in ethanol and toluene [35].

Even though SP in isopropanol has higher $\triangle A$, the fading curve became similar to SP in ethanol after $25 \mathrm{~s}$ of irradiation light removal, as shown in Figure 2(c). The change in absorbance intensities $(\triangle A)$ spectra of SP in ethanol and isopropanol decreased almost to zero at

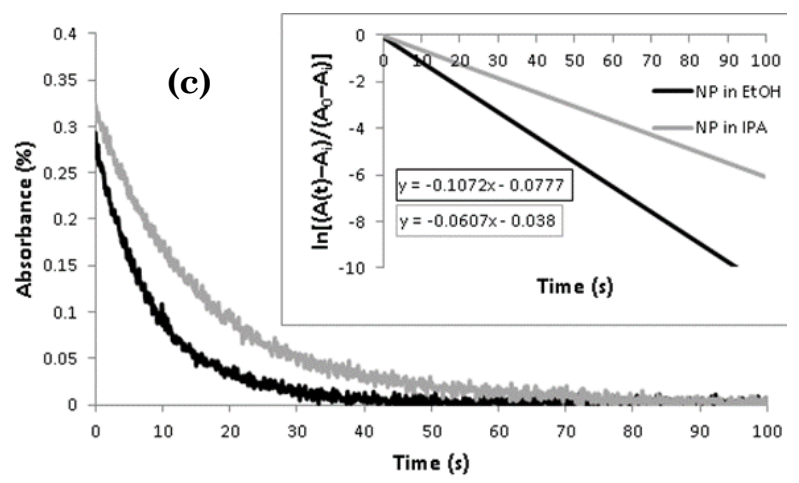

Figure 3. Fading kinetics of (a) SO in ethanol $\left(\lambda_{\max }\right.$ at $\left.613 \mathrm{~nm}\right)$ and isopropanol $\left(\lambda_{\max }\right.$ at $\left.608 \mathrm{~nm}\right)$, (b) NP in ethanol $\left(\lambda_{\max }\right.$ at $\left.430 \mathrm{~nm}\right)$ and isopropanol $\left(\lambda_{\max }\right.$ at $\left.428 \mathrm{~nm}\right)$ and (c) SP in ethanol $\left(\lambda_{\max }\right.$ at $\left.538 \mathrm{~nm}\right)$ and isopropanol $\left(\lambda_{\max }\right.$ at $\left.550 \mathrm{~nm}\right)$ after $15 \mathrm{~s}$ of UV irradiation, at $1 \mathrm{mM}$ of dye concentration. Inset: Linear plots of $\ln \left[\left(A(t)-A_{i}\right) /\left(A_{0}-A i\right)\right]$ versus time interval for the fading rate constant. 
about $175 \mathrm{~s}$. There is a moderate increase in the rate constant of SP in isopropanol $(\Delta k=$ $\left.0.0011 \mathrm{~s}^{-1}\right)$ due to stronger intermolecular interactions in less polar solvent [10]. Moreover, the polar merocyanine was stabilized in more polar solvent through hydrogen bonding with the solvent [36]. Regardless of the solvents, SO has the highest rate constant, followed by NP and SP. The same consequence has been reported when toluene was used as the solvent [24]. Comparing the dyes together with the solvents, the rate constant was greater for $\mathrm{SO}$ and NP in ethanol, while it was smaller for SP in ethanol, which means that the opened forms of SO and NP are more stable in isopropanol, whereas SP is more stable in ethanol. Equation (7) discloses the negative relationship between the fading rate constant and the half-life time. The rate constant increases as the half-life time decreases. This is because the rate constant represents the stability of the merocyanine form. If the merocyanine form is unstable, it will quickly transform back to a closed form, which results in a high rate constant and low half-life time. Furthermore, it is possible to estimate the merocyanine stability by the $\lambda_{\max }$ of the photochromic dye. The merocyanine stability was improved at shorter $\lambda_{\max }$ and thus increased the half-life time [36].

Table 1. Correlation between dye and solvent.

\begin{tabular}{lccc}
\hline & Chi-Square & $D F$ & $p$-Value \\
\hline Pearson & 0 & 2 & 1 \\
Likelihood ratio & 0 & 2 & 1 \\
\hline
\end{tabular}

3.3 Screening of Photochromic Dye and Solvent

\subsubsection{Analysis of variance (ANOVA)}

After understanding the photochromic behavior of the dyes, the next step of the investigation was to evaluate the data collected from the designed experiments. The ANOVA was used to analyze the results of the factor and level combinations. The multilevel factorial design was exploited, i.e. one variable at three levels and another variable at two levels [16]. This design was used to screen the effects of dyes and solvents on photostability and identify which dye and solvent would give the best photostability. The mean $(M(\triangle A b s))$ and standard deviation $(S D(\triangle A b s))$ of absorbance change (measured daily for 14 days) were set as the responses of interest. Natural logarithmic transformation was applied to model the variability of the response as it is in standard deviation $[16,22]$ and the natural $\log (\ln (S D(\triangle A b s)))$ was calculated.

A Pearson chi-square test and a likelihood ratio chi-square test can be exploited to determine any statistical association between the categorical independent factors. The results of both chi-square statistics in Table 1 are exactly similar. By setting the level of significance, $\alpha$, at 0.05 , the critical chi-square value is $\chi^{2} 0.05,2=$ 5.99. The null hypothesis is accepted $\left(H_{0}\right.$ : The factors are independent) because the chisquare value is less than the critical chi-square value $(0<5.99)$. Moreover, the $p$-values larger than $\alpha$ implies $(p$-value $>0.05)$ that the null hypothesis is failed to be rejected. As a result, the dye and solvent factors are independent and not associated.

Table 2. Experimental results for screening of photochromic dye and solvent. (Std $=$ standard order; Run $=$ run order).

\begin{tabular}{|c|c|c|c|c|c|c|c|c|}
\hline \multirow{3}{*}{ Replicate } & \multirow{3}{*}{ Std } & \multirow{3}{*}{ Run } & \multicolumn{4}{|c|}{ Factors } & \multicolumn{2}{|c|}{ Responses } \\
\hline & & & \multicolumn{2}{|c|}{ A: Dye } & \multicolumn{2}{|c|}{ B: Solvent } & \multirow{2}{*}{$M(\triangle A b s)$} & \multirow{2}{*}{$\ln (S D(\Delta A b s))$} \\
\hline & & & Actual & Coded & Actual & Coded & & \\
\hline 1 & 12 & 1 & SP & 3 & IPA & 2 & 0.401 & -2.34758 \\
\hline 1 & 10 & 2 & NP & 2 & IPA & 2 & 0.421 & -2.46158 \\
\hline 1 & 1 & 3 & SO & 1 & EtOH & 1 & 0.062 & -3.96859 \\
\hline 2 & 7 & 4 & SO & 1 & EtOH & 1 & 0.064 & -4.01738 \\
\hline 1 & 11 & 5 & SP & 3 & EtOH & 1 & 0.268 & -2.70456 \\
\hline 2 & 4 & 6 & $\mathrm{NP}$ & 2 & IPA & 2 & 0.413 & -2.42024 \\
\hline 1 & 9 & 7 & NP & 2 & $\mathrm{EtOH}$ & 1 & 0.353 & -2.79361 \\
\hline 2 & 5 & 8 & SP & 3 & $\mathrm{EtOH}$ & 1 & 0.254 & -2.70008 \\
\hline 2 & 6 & 9 & SP & 3 & IPA & 2 & 0.403 & -2.35599 \\
\hline 1 & 8 & 10 & SO & 1 & IPA & 2 & 0.068 & -3.78981 \\
\hline 2 & 2 & 11 & SO & 1 & IPA & 2 & 0.066 & -3.75930 \\
\hline 2 & 3 & 12 & NP & 2 & EtOH & 1 & 0.347 & -2.81008 \\
\hline
\end{tabular}


Table 2 shows the experimental results of the mean of absorbance change and the photostability of photochromic dye in solution. For the mean, NP in isopropanol has the highest mean increment of $0.421 \%$ (Run 2) whilst SO in ethanol has the lowest increase of $0.062 \%$ (Run $3)$. In terms of photostability, $\mathrm{SO}$ in ethanol gave the lowest $\ln (S D(\triangle A b s))$ value of -4.01738 (Run 4) whereas SP in isopropanol yielded the highest value of -2.34758 (Run 1 ). The low value of the natural log denotes a small standard deviation. For that reason, SO in ethanol has the smallest standard deviation while SP in isopropanol has the largest standard deviation. These statistics results were in agreement with the previous finding that the photostability of SO is better than SP [37]. SP was also shown to have poorer light fatigue in comparison to $\mathrm{SO}$ [4].

\subsubsection{Response 1: Mean of absorbance change $(M(\triangle A b s))$}

There are seven components of an ANOVA table as in Table 3, which are the source of variation (Source), degrees of freedom (DF), sequential sum of squares (Seq SS), adjusted sum of squares (Adj SS), adjusted mean squares (Adj MS), $F$-value, and $P$-value. $F$-value is the ratio of Adj MS for a source to Adj MS for the error while $P$-value is a probability that measures the evidence against the null hypothesis [16].

The critical values of $F$-distribution in Table 3 are $F_{0.05,2,6}=5.14$ and $F_{0.05,1,6}=5.99$. Since the $F$-values of dye $(4549.83>5.14)$, solvent $(583.69>5.99)$, and their interaction (183.21 > 5.14) are very high compared to the values obtained from the $F$-distribution table, the null hypothesis $\left(H_{0}: \beta=0\right)$ is rejected while the alternative hypothesis $\left(H_{1}: \beta \neq 0\right)$ is accepted, and all terms contribute significantly to the response of $M(\triangle A b s)$. The $p$-values equal to zero for these three terms are in agreement with the $F$-values. This is because the null hypothesis $\left(H_{0}: \beta=0\right)$ is also rejected when the $p$-values are smaller than $\alpha$ ( $p$-value $\leq 0.05)$. Therefore, the main effects of the dye and solvent as well as their interaction effect are statistically significant to the mean of absorbance change.

From the model summary in Table 4, the standard error of the model or regression, $S$, is 0.00506623 . This error needs to be as small as possible because it is an estimate of the amount of variability within the factors. The coefficient of determination, $R^{2}=99.94 \%$ indicates that the model can explain $99.94 \%$ of the variability in the response. The adjusted $R^{2}=$ $99.89 \%$ is also very high. A very small difference between $R^{2}$ and adjusted $R^{2}$ implies that there is a very low possibility that insignificant terms have been added in the model, deducing a high degree of model significance.

The regression coefficients for each level are tabulated in Table 5. The coefficient value is computed with respect to the reference level for the factor, which has a coefficient of zero. SP is the reference level for the dye factor and IPA is for the solvent factor, since both of them are absent from the table. Moreover, a larger coefficient is linked to a response of higher magnitude. A positive coefficient means a positive association and vice versa.

For the dye factor, SO has a higher effect on the $M(\triangle A b s)$ compared to NP and SP. The negative coefficient of SO signifies that the absorbance change in SO is lower than in SP while the positive coefficient of NP implies that NP has higher absorbance change compared to SP. From the solvent aspect, ethanol gives greater impact but yields lower $M(\triangle A b s)$ than isopropanol.

Table 4. Model summary for screening of photochromic dye and solvent (response is $M(\Delta A b s))$.

\begin{tabular}{ccl}
\hline$S$ & $R^{2}$ & $R^{2}$ (adj) \\
\hline 0.00506623 & $99.94 \%$ & $99.89 \%$ \\
\hline
\end{tabular}

Table 3. ANOVA for screening of photochromic dye and solvent (response is $M(\triangle A b s)$ ).

\begin{tabular}{lcccccc}
\hline Source & $D F$ & $S e q S S$ & $A d j S S$ & $A d j M S$ & $F$-value & $p$-value \\
\hline Dye & 2 & 0.233558 & 0.233558 & 0.116779 & 4549.83 & 0 \\
Solvent & 1 & 0.014981 & 0.014981 & 0.014981 & 583.69 & 0 \\
Dye*Solvent & 2 & 0.009405 & 0.009405 & 0.004702 & 183.21 & 0 \\
Error & 6 & 0.000154 & 0.000154 & 0.000026 & & \\
Total & 11 & 0.258098 & & & & \\
\hline
\end{tabular}


Based on the $t$-distribution table, the critical $t$-value is $t_{0.025,6}=2.447$. For each factor, the difference between the reference level and all of the terms are statistically significant except for $\mathrm{NP} * \mathrm{EtOH}$, in which the $\left|t_{0}\right|<t_{0.025,6}$ as in Table 5. This is further confirmed with the $P$ values. Since the model factors are specifically chosen, the developed statistical model is a fixed effects model. Therefore, all results are valid within the range of the parameter levels [16]. Exploiting the coefficients from Table 5, the multiple regression model relating the $M(\triangle A b s)$ with the independent variables is as in Equation (8), where $A_{1}$ is $\mathrm{SO}, A_{2}$ is NP, and $B_{1}$ is ethanol. A value of one is substituted into the model for the levels at which the response is predicted whereas zero is substituted for other levels. The values of $A_{1}, A_{2}$, and $B_{1}$ are tabulated in Table 6.

$$
\begin{aligned}
M(\Delta A b s)= & 0.26-0.195 A_{1}+0.1235 A_{2}-0.035333 B_{1} \\
& +0.033333 A_{1} B_{1}+0.0018333 A_{2} B_{1}
\end{aligned}
$$

3.3.3 Response 2: Natural log of standard deviation of absorbance change $(\ln (S D(\Delta A b s)))$

From Table 7, the critical values of $F_{0.05,2,6}=$ 5.14 and $F_{0.05,1,6}=5.99$ are the same as Table 3, as the values of DF and $\alpha$ are the same. Since the $F$-values of dye $(5117.76>5.14)$, solvent $(642.63>5.99)$, and their interaction (14.07 > 5.14) are larger than the critical values, the null hypothesis $\left(H_{0}: \beta=0\right)$ is rejected, while the alternative hypothesis $\left(H_{1}: \beta \neq 0\right)$ is accepted, which implies that all terms have a significant impact on photostability. The $p$-values smaller than $\alpha$ are also in accordance with the $F$ values, which result in the rejection of the null hypothesis $\left(H_{0}: \beta=0\right)$. Hence, from the ANO$\mathrm{VA}$, it is found that the $\ln (S D(\Delta A b s))$ is significantly affected by the dye, solvent, and dyesolvent interaction.

The small value of $S(0.0211786)$ in Table 8 denotes small variability within the factors. Moreover, $R^{2}$ suggests that the model can describe $99.95 \%$ of the response variability. The adjusted $R^{2}(99.90 \%)$ is also very high. This

Table 5. Estimated regression coefficients for screening of photochromic dye and solvent (response is $M(\triangle A b s))$.

\begin{tabular}{lccccc}
\hline & Term & Coefficient & $S E$ Coefficient & $T$-value & $p$-value \\
\hline Dye & Constant & 0.260000 & 0.001462 & 177.78 & 0 \\
& $\mathrm{SO}$ & -0.195000 & 0.002068 & -94.28 & 0 \\
\hline Solvent & $\mathrm{NP}$ & 0.123500 & 0.002068 & 59.71 & 0 \\
\hline Dye* Solvent & $\mathrm{EtOH}$ & -0.035333 & 0.001462 & -24.16 & 0 \\
& $\mathrm{SO}$ EtOH & 0.033333 & 0.002068 & 16.12 & 0 \\
& $\mathrm{NP} \mathrm{EtOH}$ & 0.001833 & 0.002068 & 0.89 & 0.41 \\
\hline
\end{tabular}

Table 6. Values of $A_{1}, A_{2}$, and $B_{1}$ in Equations (8) and (9).

\begin{tabular}{ccccccc}
\hline Dye & Solvent & $A_{1}$ & $A_{2}$ & $B_{1}$ & $A_{1} B_{1}$ & $A_{2} B_{1}$ \\
\hline SO & EtOH & 1 & 0 & 1 & 1 & 0 \\
NP & EtOH & 0 & 1 & 1 & 0 & 1 \\
SP & EtOH & -1 & -1 & 1 & -1 & -1 \\
SO & IPA & 1 & 0 & -1 & -1 & 0 \\
NP & IPA & 0 & 1 & -1 & 0 & -1 \\
SP & IPA & -1 & -1 & -1 & 1 & 1 \\
\hline
\end{tabular}

Table 7. ANOVA for screening of photochromic dye and solvent (response is $\ln (S D(\triangle A b s))$ ).

\begin{tabular}{ccccccc}
\hline Source & $D F$ & $S e q S S$ & $A d j S S$ & $A d j M S$ & $F$-value & $p$-value \\
\hline Dye & 2 & 4.59095 & 4.59095 & 2.29548 & 5117.76 & 0 \\
Solvent & 1 & 0.28824 & 0.28824 & 0.28824 & 642.63 & 0 \\
Dye*Solvent & 2 & 0.01262 & 0.01262 & 0.00631 & 14.07 & 0.005 \\
Error & 6 & 0.00269 & 0.00269 & 0.00045 & & \\
Total & 11 & 4.8945 & & & & \\
\hline
\end{tabular}


happens because all the terms in the model are statistically significant.

Table 9 presents the regression coefficients for each factor level. The lower the value of $\ln (S D(\triangle A b s))$, the better the photostability. Considering dye as the factor, SO produces the best photostability because of the negative sign. Also, the photostability was improved when ethanol is used as the solvent. From Table 9, the critical value of $t$ is $t_{0.025,6}=2.447$, which is identical to the value in Table 5 since the DF and $\alpha$ values are as before. All of the terms have significant difference when compared to the reference level because the lowest $\left|t_{0}\right|$ is 2.95 , still larger than 2.447 . These $T$-values are parallel with the $P$-values because all of the $P$ values are smaller than 0.05 .

Table 8. Model summary for screening of photochromic dye and solvent (response is $\ln (S D(\Delta A b s)))$.

\begin{tabular}{ccc}
\hline$S$ & $R^{2}$ & $R^{2}(\mathrm{adj})$ \\
\hline 0.0211786 & $99.95 \%$ & $99.90 \%$ \\
\hline
\end{tabular}

The developed multiple regression equation for $\ln (S D(\triangle A b s))$ from the coefficients in Table 9 is as in Equation (9) where $A_{1}$ is $\mathrm{SO}, A_{2}$ is $\mathrm{NP}$, and $B_{1}$ is ethanol. The values of $A_{1}, A_{2}$, and $B_{1}$ are listed in Table 6.

$$
\begin{aligned}
\ln (S D(\triangle A b s))= & -3.01073-0.873037 A_{1}+0.389356 A_{2} \\
& -0.154983 B_{1}+0.045768 A_{1} B_{1} \\
& -0.025484 A_{2} B_{1}
\end{aligned}
$$

\subsection{Model Adequacy Checking}

\subsubsection{Response 1: Mean of absorbance change} $(M(\triangle A b s))$

There are three residual plots that can be employed to validate the model assumptions. All of these plots were plotted using the standardized residuals in order to detect any outlier simultaneously. The first one is the normal probability plot of the standardized residuals as in Figure 4(a). The normal probability plot is exploited instead of a histogram because this plot is more effective in interpreting small samples of less than 200. The residuals for $M(\triangle A b s)$ are normally distributed since the plot resembles a straight line, with the points

Table 9. Estimated regression coefficients for screening of photochromic dye and solvent (response is $\ln (S D(\triangle A b s)))$.

\begin{tabular}{lccccc}
\hline & Term & Coefficient & $S E$ Coefficient & $T$-value & $p$-value \\
\hline Dye & Constant & -3.010730 & 0.006110 & -492.45 & 0 \\
& $\mathrm{SO}$ & -0.873037 & 0.008646 & -100.97 & 0 \\
\hline Solvent & $\mathrm{NP}$ & 0.389356 & 0.008646 & 45.03 & 0 \\
\hline Dye*Solvent & $\mathrm{EtOH}$ & -0.154983 & 0.006114 & -25.35 & 0 \\
& $\mathrm{SO} * \mathrm{EtOH}$ & 0.045768 & 0.008646 & 5.29 & 0.002 \\
& $\mathrm{NP} \mathrm{EtOH}$ & -0.025484 & 0.008646 & -2.95 & 0.026 \\
\hline
\end{tabular}

Table 10. Grouping information of dye and solvent for $M(\triangle A b s)$ for $\ln (S D(\Delta A b s))$.

\begin{tabular}{lcccc}
\hline & Dye/Solvent & $N$ & Mean & Grouping \\
\hline Dye for $M(\triangle A b s)$ & NP & 4 & 0.3835 & $A$ \\
& SP & 4 & 0.3315 & $B$ \\
& SO & 4 & 0.065 & $C$ \\
\hline Solvent for $M(\triangle A b s)$ & IPA & 6 & 0.29533 & $A$ \\
& EtOH & 6 & 0.22467 & $B$ \\
\hline Dye for $\ln (S D(\Delta A b s))$ & SP & 4 & -2.527 & $A$ \\
& NP & 4 & -2.621 & $B$ \\
\hline Solvent for $\ln (S D(\Delta A b s))$ & SO & 4 & -3.884 & $C$ \\
& IPA & 6 & -2.856 & $A$ \\
\hline
\end{tabular}


generally falling close to the line. This is consistent with the Anderson-Darling test, as the $p$-value is 0.824 ( $p$-value $>0.05$ ).

For the model validation, both plots of the standardized residuals versus fitted values (Figure 4(b)) and the standardized residuals versus run order (Figure 4(c)) reveal no clear pattern and the points are randomly distributed around zero. Hence, both constant variance and independence assumptions for $M(\triangle A b s)$ are met and verified. These three residual plots signify no violations of assumptions. The ANOVA model fits the data sufficiently well. Addi-
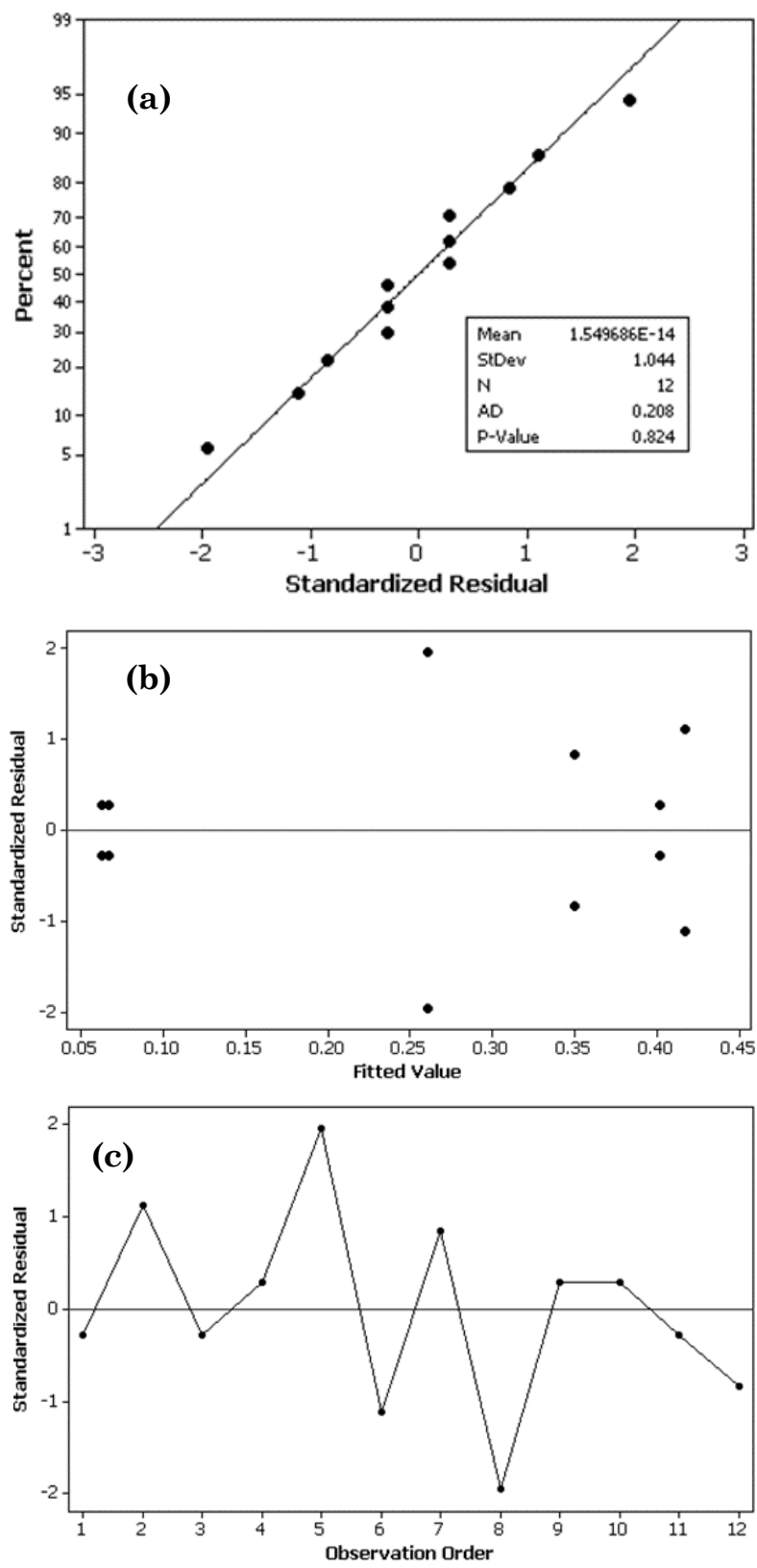

Figure 4. (a) Normal probability plot of standardized residuals for $M(\triangle A b s)$. Standardized residuals vs (b) fits and (c) order for $M(\triangle A b s)$. tionally, there is no outlier observed in these plots since the standardized residual values are within \pm 2 .

Tukey's multiple comparison test is employed to compare all possible pairs of factors and determine which pairs of factor means are significantly different from each other. Table 10 reveals the grouping information of the dye and solvent, with $M(\Delta A b s)$ and $\ln (S D(\triangle A b s))$ being the response. The NP, SP, and SO means are significantly different from each other because they do not share a grouping letter. NP dye has the biggest mean of $M(\Delta A b s)$ whilst SO has the smallest mean. The Tukey's test dis-
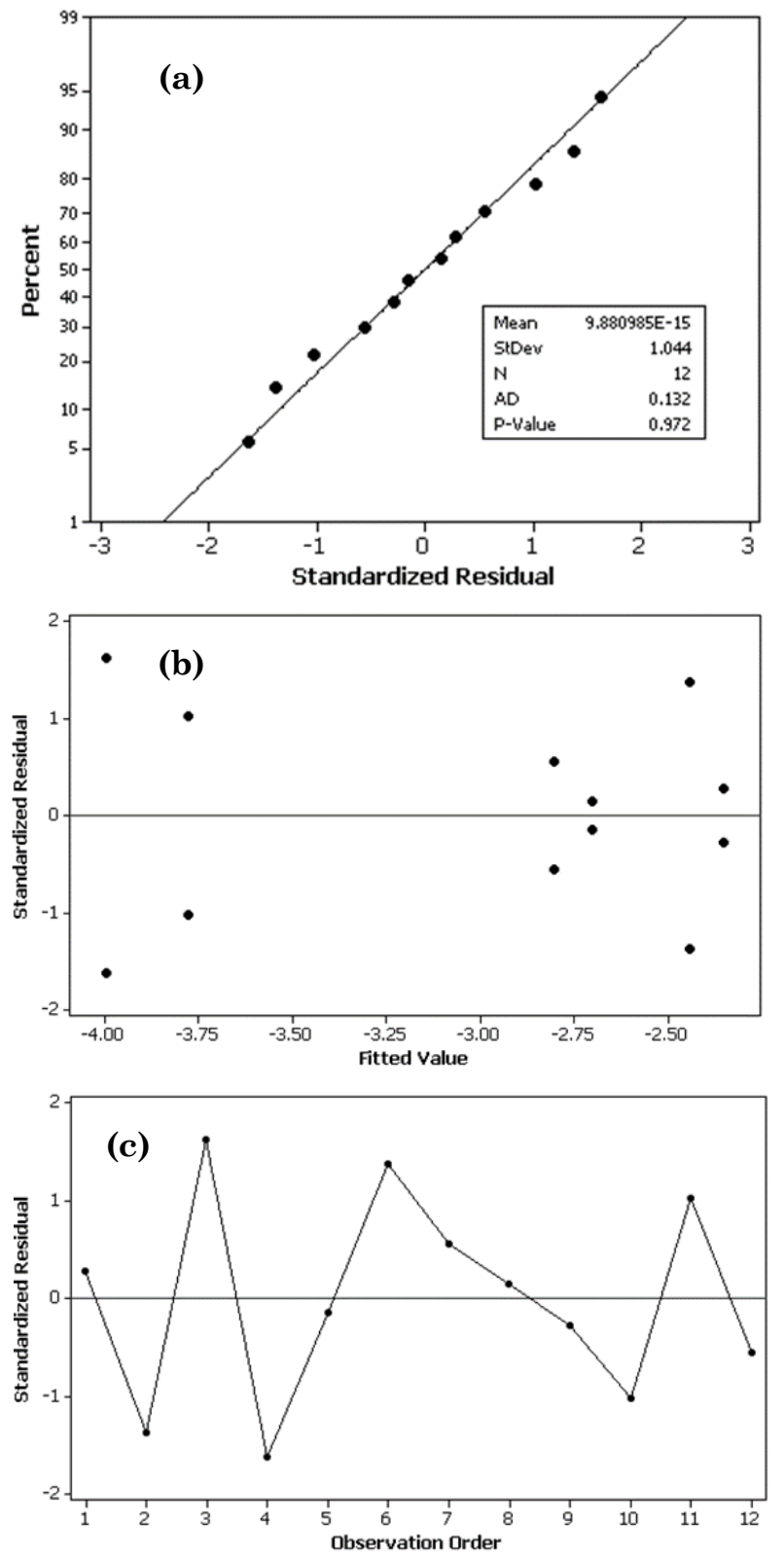

Figure 5. (a) Normal probability plot of standardized residuals for $\ln (S D(\Delta A b s))$. Standardized residuals vs (b) fits and (c) order for $\ln (S D(\Delta A b s))$. 
closes that the means of $M(\triangle A b s)$ of isopropanol and ethanol were significantly different average. Ethanol exhibited smaller $M(\triangle A b s)$ than isopropanol.

3.4.2 Response 2: Natural log of standard deviation of absorbance change $(\ln (S D(\triangle A b s)))$

Figure 5(a) displays the normal probability plot of the standardized residuals for $\ln (S D(\Delta A b s))$. All of the points fall nearly on the straight line and thus fulfil the normality condition. The $p$-value of the Anderson-Darling test of 0.972 justifies the normality.

The other two plots utilized for validation are shown in Figure 5(b-c). The assumptions of constant variance and independence for $\ln (S D(\Delta A b s))$ are proven since the points are vertically spread in these two plots of the standardized residuals versus fitted values (Figure 5(b)) and the standardized residuals versus run order (Figure 5(c)). The ANOVA model fits the data well enough because all of the residual plots do not indicate a violation of assumptions. Besides, the range of standardized residual values from -2 to +2 shows nonappearance of outlier.

Table 10 displays the grouping information of the dye for $\ln (S D(\Delta A b s))$ response. The means of these three dyes have significantly different average $\ln (S D(\Delta A b s))$. SO dye has the lowest mean of $\ln (S D(\triangle A b s))$ which implies that $\mathrm{SO}$ is the most stable dye as compared to NP and SP. The means of $\ln (S D(\triangle A b s))$ for isopropanol and ethanol indicates that the significantly different average of $\ln (S D(\triangle A b s))$ with ethanol produces lower $\ln (S D(\triangle A b s))$ than isopropanol. The molecules of spironaphthoxazine (SNO), which is an SO, in ethanol solvent is excited to produce the hydrogen bonded species through $\mathrm{C}-\mathrm{O}$ bond breakage during the relaxation process of the excited states [38]. Unlike ethanol which is a protic solvent of high polarity, propanol is a lower polarity solvent [38].

\section{Conclusions}

In solution form, all the three types of photochromic dyes, which are spirooxazine, naphthopyran, and spiropyran, exhibited positive or normal photochromism. The absorption peak was detected at $608 \mathrm{~nm}$ for isopropanol and 613 $\mathrm{nm}$ for ethanol, which designates that SO shows positive solvatochromism. NP dye also unveils positive solvatochromism because the maximum absorption band moved bathochromically from $428 \mathrm{~nm}$ to $430 \mathrm{~nm}$ as the solvent polarity increases. SP however exhibits negative solvatochromism as the maximum wave- length was shifted to shorter wavelength in more polar solvent, from $550 \mathrm{~nm}$ in isopropanol to $538 \mathrm{~nm}$ in ethanol. The analysis of multilevel factorial design reveals that the main factors of dye and solvent, and also their interaction gave statistically significant impact on the change in absorbance intensities and photostability. Naphthopyran has the highest mean absorbance than spiropyran and spirooxazine. Nevertheless, spirooxazine is the most photostable dye, followed by naphthopyran and spiropyran. All these dyes have better photostability in ethanol compared to isopropanol.

\section{Acknowledgments}

This research was supported by the Ministry of Higher Education under Fundamental Research Grant Scheme (FRGS Grant No. 11029-0177). Also, the authors would like to thank Noor Zalikha Mohamed Islam for her useful assistance.

\section{References}

[1] Dürr, H. (2003). Chapter 1 - General Introduction, in H. Dürr, H. Bouas-Laurent (Editors) Photochromism. Amsterdam: Elsevier Science. DOI: 10.1016/B978-0444513229/50005-1.

[2] Nigel Corns, S., Partington, S.M., Towns, A.D. (2009). Industrial organic photochromic dyes. Coloration Technology, 125(5), 249-261. DOI: $10.1111 / \mathrm{j} .1478-4408.2009 .00204 . x$.

[3] Cheng, H., Yoon, J., Tian, H. (2018). Recent advances in the use of photochromic dyes for photocontrol in biomedicine. Coordination Chemistry Reviews, 372, 66-84. DOI: 10.1016/j.ccr.2018.06.003.

[4] Xia, H., Xie, K., Zou, G. (2017). Advances in spiropyrans/spirooxazines and applications based on Fluorescence Resonance Energy Transfer (FRET) with fluorescent materials. Molecules, 22(12), 2236 . DOI: $10.3390 /$ molecules22122236.

[5] Xia, H., Li, J., Zou, G., Zhang, Q., Jia, C. (2013). A highly sensitive and reusable cyanide anion sensor based on spiropyran functionalized polydiacetylene vesicular receptors. Journal of Materials Chemistry A, 1(36), 10713-10719. DOI: 10.1039/C3TA11526G.

[6] Sahoo, P.R., Kumar, S. (2016). Photochromic spirooxazine as highly sensitive and selective probe for optical detection of $\mathrm{Fe} 3+$ in aqueous solution. Sensors and Actuators B: Chemical, 226, 548-552. DOI: 10.1016/j.snb.2015.12.039

[7] Little, A.F., Christie, R.M. (2016). Textile applications of commercial photochromic dyes. 
Part 6: photochromic polypropylene fibres. Coloration Technology, 132(4), 304-309. DOI: 10.1111/cote.12221.

[8] Nakazumi, H., Makita, K., Nagashiro, R. (1997). New sol-gel photochromic thin films made by super-fine particles of organic photochromic compounds. Journal of Sol-Gel Science and Technology, 8(1-3), 901-909. DOI: 10.1023/A:1018331401548.

[9] Hou, L., Mennig, M., Schmidt, H.K. (1994). Improvement of photofatigue resistance of spirooxazine entrapped in organic-inorganic composite synthesized via the sol-gel process. In Proc. SPIE Sol-Gel Optics III, 2288, 328339. San Diego, CA, United States (13 October 1994). DOI: 10.1117/12.188966.

[10] Malatesta, V. (2002). Photodegradation of Organic Photochromes. In J.C. Crano, R.J. Guglielmetti (Editors) Organic Photochromic and Thermochromic Compounds: Volume 2: Physicochemical Studies, Biological Applications, and Thermochromism. Boston, MA: Springer US. DOI: 10.1007/0-306-46912-x_3.

[11] Islam, N.Z.M., Nazri, S.A.A.A., Nadir, N., Zainuddin, M.T. (2017). The study of photochromic performance and photofatigue behavior of spirooxazine. AIP Conference Proceedings, 1901 (1), 130014 . DOI: 10.1063/1.5010574.

[12] Alimi, F., Boubakri, A., Tlili, M.M., Ben Amor, M. (2014). A comprehensive factorial design study of variables affecting $\mathrm{CaCO}_{3}$ scaling under magnetic water treatment. $W a-$ ter Science and Technology, 70(8), 1355-1362. DOI: $10.2166 /$ wst.2014.377.

[13] del Campo, G., Gallego, B., Berregi, I. (2006). Fluorimetric determination of histamine in wine and cider by using an anion-exchange column-FIA system and factorial design study. Talanta, 68(4), 1126-1134. DOI: 10.1016/j.talanta.2005.07.019.

[14] Krishnan, J., Kishore, A.A., Suresh, A., Madhumeetha, B., Prakash, D.G. (2017). Effect of $\mathrm{pH}$, inoculum dose and initial dye concentration on the removal of azo dye mixture under aerobic conditions. International Biodeterioration \& Biodegradation, 119, 16-27. DOI: 10.1016/j.ibiod.2016.11.024.

[15] ReVelle, J.B., Margetts, D.N. (2009). Home Builder's Guide to Continuous Improvement: Schedule, Quality, Customer Satisfaction, Cost, and Safety. Boca Raton: CRC Press.

[16] Montgomery, D.C. (2005). Design and analysis of experiments. Hoboken, NJ: John Wiley \& Sons.

[17] Nadir, N., Wahid, Z., Shafie, A., Muthalif, A.A., Malek, M.A., Aziz, N.N.A., Zainuddin, M., Islam, N.M. (2013). Development of real time experimental system for investigating photochromic response to UV irradiation. IOP Conference Series: Materials Science and Engineering, 53, 012083. DOI: 10.1088/1757899X/53/1/012083.

[18] Nadir, N., Wahid, Z., Zainuddin, M.T., Islam, M., Zalikha, N. (2014). Photochromic Behavior of Spiropyrans: The Effect of Substituent. Advanced Materials Research, 925, 323-328. DOI:10.4028/www.scientific.net/AMR.925.323

[19] Negri, R.M., Prypsztejn, H.E. (2001). An experiment on photochromism and kinetics for the undergraduate laboratory. Journal of Chemical Education, 78(5), 645. DOI: 10.1021/ed078p645.

[20] Feczkó, T., Kovács, M., Voncina, B. (2012). Improvement of fatigue resistance of spirooxazine in ethyl cellulose and poly (methyl methacrylate) nanoparticles using a hindered amine light stabilizer. Journal of Photochemistry and Photobiology A: Chemistry, 247, 17. DOI: 10.1016/j.jphotochem.2012.08.001.

[21] Zhang, S., Zhang, Q., Ye, B., Li, X., Zhang, X., Deng, Y. (2009). Photochromism of spiropyran in ionic liquids: enhanced fluorescence and delayed thermal reversion. The Journal of Physical Chemistry B, 113(17), 6012-6019. DOI: $10.1021 /$ jp9004218.

[22] Mathews, P.G. (2005). Design of Experiments with MINITAB. Milwaukee: ASQ Quality Press.

[23] Lesik, S.A. (2018). Applied Statistical Inference with MINITAB ${ }^{\circledR}$. London: Chapman and Hall/CRC.

[24] Bouas-Laurent, H., Dürr, H. (2001). Organic photochromism (IUPAC technical report). Pure and Applied Chemistry, 73(4), 639-665. DOI: $10.1351 /$ pac200173040639.

[25] Levy, D. (1997). Recent applications of photochromic sol-gel materials. Molecular Crystals and Liquid Crystals Science and Technology. Section A. Molecular Crystals and Liquid Crystals, 297(1), 31-39. DOI: 10.1080/10587259708036100.

[26] Lafuma, A., Chodorowski-Kimmes, S., Quinn, F.X., Sanchez, C. (2003). Photochromic properties of a spirooxazine and a spiropyran in alcoholic solutions of zirconium and aluminium alkoxides: influence of the ethyl acetoacetate chelating agent on the optical properties. European Journal of Inorganic Chemistry, $2003(2), \quad 331-338$. D O I : 10.1002/ejic.200390045.

[27] Salemi-Delvaux, C., Luccioni-Houze, B., Baillet, G., Giusti, G., Guglielmetti, R. (1995). Effect of photodegradation on the thermal bleaching rate constant of photochromic compounds in spiro [indoline-pyran] and spiro 
[indoline-oxazine] series. Journal of Photochemistry and Photobiology A: Chemistry, 91(3), 223-232. DOI: 10.1016/10106030(95)04113-X.

[28] Harbron, E.J., Davis, C.M., Campbell, J.K., Allred, R.M., Kovary, M.T., Economou, N.J. (2009). Photochromic dye-doped conjugated polymer nanoparticles: photomodulated emission and nanoenvironmental characterization. The Journal of Physical Chemistry C, 113(31), 13707-13714. DOI: 10.1021/jp9037864.

[29] Zayat, M., Levy, D. (2003). Photochromic naphthopyrans in sol-gel ormosil coatings. Journal of Materials Chemistry, 13(4), 727730. DOI: 10.1039/B211759B.

[30] Keum, S.-R., Hur, M.-S., Kazmaier, P.M., Buncel, E. (1991). Thermo-and photochromic dyes: indolino-benzospiropyrans. Part 1 . UVVIS spectroscopic studies of 1, 3, 3-spiro (2 H1-benzopyran-2, 2'-indolines) and the openchain merocyanine forms; solvatochromism and medium effects on spiro ring formation. Canadian Journal of Chemistry, 69(12), 1940-1947. DOI: 10.1139/v91-279.

[31] Kopelman, R.A., Snyder, S.M., Frank, N.L. (2003). Tunable photochromism of spirooxazines via metal coordination. Journal of the American Chemical Society, 125(45), 1368413685. DOI: $10.1021 /$ ja036306y

[32] Piech, M., Bell, N.S. (2006). Controlled synthesis of photochromic polymer brushes by atom transfer radical polymerization. Macromolecules, 39(3), 915-922. DOI: 10.1021/ma0512760.

[33] Such, G., Evans, R.A., Yee, L.H., Davis, T.P. (2003). Factors influencing photochromism of spiro-compounds within polymeric matrices. Journal of Macromolecular Science, Part C: Polymer Reviews, 43(4), 547-579. DOI: 10.1081/MC-120025978.

[34] McGarvey, D.J. (2006). Industry-linked context-based chemistry practicals. New Directions in the Teaching of Physical Sciences, (2), 57-64. DOI: 10.29311/ndtps.v0i2.436.
[35] Luccioni-Houzé, B., Campredon, M., Guglielmetti, R., Giusti, G. (1997). Kinetic analysis of fluoro- $[2 \mathrm{H}]$-chromenes at the photostationary states. Molecular Crystals and Liquid Crystals Science and Technology. Section A. Molecular Crystals and Liquid Crystals, 297 ( 1 ), $\quad 161-165$. D O I : $10.1080 / 10587259708036117$

[36] Shumburo, A., Biewer, M.C. (2002). Stabilization of an organic photochromic material by incorporation in an organogel. Chemistry of Materials, 14(9), 3745-3750. DOI: 10.1021/cm020421a.

[37] Crano, J.C., Guglielmetti, R.J. (2002). Introduction. In J.C. Crano, R.J. Guglielmetti (Editors) Organic Photochromic and Thermochromic Compounds: Volume 2: Physicochemical Studies, Biological Applications, and Thermochromism. Boston, MA: Springer US. DOI: 10.1007/0-306-46912-x_1.

[38] Nishikiori, H., Tanaka, N., Takagi, K., Fujii, T. (2003). Solvent effect on fluorescence spectra of a spirooxazine. Research on Chemical Intermediates, 29(5), 485-493. DOI: $10.1163 / 156856703322149026$.

[39] Pardo, R., Zayat, M., Levy, D. (2008). Effect of the chemical environment on the lightinduced degradation of a photochromic dye in ormosil thin films. Journal of Photochemistry and Photobiology A: Chemistry, 198(2), 232236. DOI: 10.1016/j.jphotochem.2008.03.013.

[40] Piard, J. (2014). Influence of the Solvent on the Thermal Back Reaction of One Spiropyran. Journal of Chemical Education, 91(12), 2105-2111. DOI: 10.1021/ed4005003.

[41] Rezvanova, A.A., Frolova, L.A., Troshin, P.A. (2016). Design of optical memory elements based on n-type organic field-effect transistors comprising a light-sensitive spirooxazine layer. Mendeleev Communications, 26(1), 2628. DOI: 10.1016/j.mencom.2016.01.011.

Selected and Revised Papers from International Conference on Sustainable Energy and Catalysis 2021 (ICSEC 2021) (https://engineering.utm.my/chemicalenergy/icsec2021/) (School of Chemical and Energy Engineering, Faculty of Engineering, Universiti Teknologi Malaysia, 16-17th February 2021) after Peer-reviewed by Scientific Committee of ICSEC 2021 and Peer-Reviewers of Bulletin of Chemical Reaction Engineering \& Catalysis. Editors (Guest) in this ICSEC 2021 section are Nor Aishah Saidina Amin, Mohd Asmadi Mohammed Yussuf, Salman Raza Naqvi, while Editor in Chief is I. Istadi. 